\title{
Application of Hydrothermal Reaction to Biodegradability Improvement of Refractory Pollutants: Structural Conversion of Di- and Trichloroacetic Acid to Biodegradable Products
}

\author{
Kyoungrean $\mathrm{KIM}^{1 *}$, Masafumi FUJITA ${ }^{2}$, Hiroyuki DAIMON ${ }^{1}$ and Koichi FUJIE ${ }^{1}$ \\ ${ }^{1}$ Department of Ecological Engineering, Toyohashi University of Technology, \\ Hibarigaoka 1-1, Tempaku-cho, Toyohashi, Aichi 441-8580, JAPAN \\ Tel.\&Fax.: +81-532-44-6910, E-mail: kim@fujielab.eco.tut.ac.jp \\ ${ }^{2}$ Department of Civil and Environmental Engineering, University of Yamanashi, \\ 4-3-11 Takeda, Kofu, Yamanashi 400-8511, JAPAN
}

\begin{abstract}
Biodegradability improvement of refractory pollutants by hydrothermal reaction was investigated based on their structural conversion. Di- and Trichloroacetic acid were used as test sample, representing linear hydrocarbon structured refractory pollutants. At $250{ }^{\circ} \mathrm{C}$ and $4 \mathrm{MPa}$, attached recalcitrant chlorine atoms were eliminated by hydrolysis at the beginning of hydrothermal reaction. Biodegradable organic acids were yielded from hydrolyzed intermediates by dehydration and thermal decomposition. The decomposition rates of chloroacetic acids increased with increasing the number of attached chlorine atoms. During the initial structural conversion by hydrothermal reaction, the reduction of carbon contents of dichloroacetic acid did not exceed $6 \%$ under the tested conditions. The similar results, however, were not observed in case of trichloroacetic acid. Biodegradable products were reduced by thermal decomposition as reaction time increased. The biodegradability of reaction products was not fatally retarded despite the presence of chlorine ions under the tested conditions. Hydrothermal reaction was proved as suitable pretreatment method to obtain biodegradable products from the structural conversion of refractory pollutants such as chloroacetic acids for the following biological treatment methods.
\end{abstract}

Keywords: Hydrothermal reaction, refractory pollutants, structural conversion, biodegradability improvement, chloroacetic acids

\section{INTRODUCTION}

Generally, chlorinated compounds are considered as refractory pollutants due to inherent recalcitrant chlorine atoms [1]. Chloroacetic acids (mono-, di- and trichloroacetic acid; CAAs) are often discharged from the manufacture of drug, dyes and chemicals as intermediates [2, 3]. Because of their low acute toxicity, high solubility in water and low volatility, CAAs are toxic to plant, alga and animal $[4,5]$ and resist biodegradation during extremely long time periods [6]. To solve these problems, biodegradation of refractory pollutants has been continuously researched, because it is important to understand the basic and ultimate fate in aquatic and terrestrial ecosystems [7]. Advanced treatment methods based on chemical oxidation or mineralization cannot always secure constant effluent water qualities, corresponding to the fluctuation of wastewater. Conventional biological treatment methods, such as activated sludge process are retarded by refractory pollutants. Hence, a new pretreatment method is required to obtain biodegradable products from refractory pollutants, without much reduction of carbon contents for the following conventional biological treatment methods. The desired process is not just complete decomposition, but structural conversion to easily degradable substances as the results of partial destruction of refractory pollutants.

Hydrothermal reaction has been attracting many researchers, because of the fascinating characteristics of water as reaction medium at elevated temperatures and pressures $[8,9]$. In our previous research, we reported the possibility of biodegradability improvement of poly vinyl alcohol (PVA) in $10 \mathrm{~min}$ 
by hydrothermal reaction [10], structural conversion and reaction mechanism of monochloroacetic acid (MCAA) under hydrothermal conditions [11]. However, more extended investigation is required to clarify the reaction mechanism of various refractory pollutants, including other kinds of CAAs, under hydrothermal conditions and to apply this technology to real wastewater.

This research is focused on the structural conversion of di- and trichloroacetic acid (DCAA, TCAA) under hydrothermal conditions. Under these conditions, hydrolysis, thermal decomposition and catalytic effect of water could remove attached recalcitrant chlorine atoms in short reaction time. This process could likely improve biodegradability of CAAs regardless of the number of attached chlorine atoms. The purposes of this research are to investigate the effect of the number of attached chlorine atoms on the reaction mechanism of CAAs under hydrothermal conditions and to clarify the relationship between products obtained from the initial structural conversion of CAAs and their biodegradability change. Besides, the application of hydrothermal reaction as a pretreatment method of refractory pollutants is also investigated for the following conventional biological treatment methods.

\section{MATERIALS AND METHODS}

\subsection{Reagent}

DCAA, TCAA and other organic acids (Malic, Glycolic, Citric and Formic acid) were obtained from Tokyo Chemical Industry Co. (Tokyo, Japan). All chemicals were guaranteed grade. Malic, glycolic, citric and formic acid were used as standard materials for qualitative and quantitative analysis of products obtained from CAAs.

\subsection{Batch reactor apparatus}

Reaction was carried out using a batch reactor apparatus (TSC-006, Taiatsu Glass Corp.). It mainly consists of a stirrer, a pressure gauge, a reactor and a molten salt bath containing mixture of potassium nitrate and sodium nitrate $[\mathbf{1 2}, \mathbf{1 3}]$. Two batch reactors were used in this study. One batch reactor (reactor 1), made up of hastelloy C22 (Ni, Cr, Mo alloy), has a total inner volume of $65.9 \mathrm{~cm}^{3}$. The other reactor (reactor 2), made by stainless steel (sus 316), has an effective inner volume of $5.9 \mathrm{~cm}^{3}$. The maximum operational conditions of both reactors are $450{ }^{\circ} \mathrm{C}$ and $45 \mathrm{MPa}$. Reactor 1 takes 9 min to reach $250{ }^{\circ} \mathrm{C}$ starting at room temperature. While, reactor 2 requires $1.5 \mathrm{~min}$ to reach the same temperature. The heat-up time depends on the desired reaction temperatures $[\mathbf{1 1}, \mathbf{1 2}]$. Reactor 2 was only used to investigate reaction rates within 9 min, because of its short heat-up time.

\subsection{Experimental methodologies}

The sample concentration of DCAA and TCAA were adjusted to 4.16 and $4.17 \mathrm{mM}$ with distilled water, respectively. In order to investigate short time reaction of highly concentrated refractory pollutants by hydrothermal reaction, higher concentration range than that of real wastewater stream was selected. The reactions of DCAA and TCAA with water were conducted at $250{ }^{\circ} \mathrm{C}$ and $4 \mathrm{MPa}$ within $60 \mathrm{~min}$. The desired pressure was obtained by adjusting the initial sample volume using the data on steam table. During the experiments, reaction pressure was verified using the pressure gauge attached the batch reactor apparatus. In each experiment, sample was placed into the reactor. The reactor was sealed, and then the air inside was replaced with pure nitrogen gas. The reactor was put into the preheated molten salt bath during the desired reaction time. Then the reactor was immediately quenched in water bath, effectively ceasing any occurring reactions. In order to check reproducibility of data, every experiment was conducted at three times under the same reaction conditions.

\subsection{Analytical methods}

DCAA and TCAA were determined by gas chromatography (HP6890 GC, Hewlett-Packard Co.) coupled with mass selective detector (HP5973 MSD, Hewlett-Packard Co.). The sample was prepared for GC analysis following the method reported by Xie [14]. A $3 \mu \mathrm{L}$ of sample was injected into GC with splitless mode and separated in a capillary column (HP-5MS, $30 \mathrm{~m} \times 0.25 \mathrm{~mm}$ i.d., $0.25 \mu \mathrm{m}$ d.f., HewlettPackard Co.). The flow rate of carrier gas was $1.0 \mathrm{~mL} / \mathrm{min}$ at constant flow mode. The oven temperature was held isothermally at $40{ }^{\circ} \mathrm{C}$ for $3 \mathrm{~min}$, then ramped to $200{ }^{\circ} \mathrm{C}$ at the rate of $10{ }^{\circ} \mathrm{C} / \mathrm{min}$ and held for $1 \mathrm{~min}$. The injector temperature was $200{ }^{\circ} \mathrm{C}$ and the transfer line was maintained at $280{ }^{\circ} \mathrm{C}$. The mass selective 
detector was operated in the electron impact (EI) mode. The temperature of ion source was $230{ }^{\circ} \mathrm{C}$ and the electron energy was $70 \mathrm{eV}$. For the investigation of reaction mechanism, reaction products were also analyzed using an organic acid analyzer (LC-10A, Shimadzu Corp.), with two ion-exclusion columns (Shimpark SCR-102H, Shimadzu Corp.) connected in series and an electroconductivity detector (CDD-6A, Shimadzu Corp.). The chlorine ions of products were determined using an ion chromatography analyzer (DX-120, DIONEX Corp.). It consists of an analytical column (Ionpac AS14 P/N 46124, DIONEX Corp.), a guard column (Ionpac AS14 P/N 46134, DIONEX Corp.) and a conductivity detector (DS4, DIONEX Corp.) supported by self-regenerating suppressor (ASAR P/N 53946, DIONEX Corp.). In order to evaluate the change of water quality indexes, following items were analyzed before and after reactions. Total organic carbon (TOC) and dissolved organic carbon (DOC) were measured using a TOC analyzer (TOC-5000A, Shimadzu Corp.). Chemical oxygen demand $\left(\mathrm{COD}_{\mathrm{Cr}}\right)$ was analyzed using a $\mathrm{COD}$ analyzer, consisted by a COD reactor ( $\mathrm{P} / \mathrm{N}$ 45600-00, HACH Corp.) and a spectrophotometer (DR/3000, HACH Corp.). Biochemical oxygen demand (BOD) was measured using a BOD tester (BOD Tester 200F, Taitec Corp.). In the beginning of BOD analysis, all samples were adjusted to the $\mathrm{pH}$ value of 7 . The $\mathrm{pH}$ was measured by using $\mathrm{pH}$ meter (F-23, HORIBA). Before analysis of organic acid, chlorine ion and DOC, all samples were filtered to separate solid materials, using micro-syringes and filters with pore size of $0.45 \mu \mathrm{m}$.

\section{RESULTS AND DISCUSSION}

\subsection{Product evaluation}

Reaction was conducted at $250{ }^{\circ} \mathrm{C}$ and $4 \mathrm{MPa}$. From our previous research [11], mineralization was less than $3 \%$ followed by structural conversion from MCAA to biodegradable organic acid under the reaction conditions. The heat-up state of reactor from room temperature to $90 \%$ of each reaction temperature was defined as transition state. The reaction times were also defined as the elapsed time from the transition state.

Reaction products are evaluated in this section to know the reaction mechanism of DCAA and TCAA under hydrothermal conditions. Figure 1 shows the effect of reaction time on the content change of products from DCAA and TCAA by hydrothermal reaction at $250{ }^{\circ} \mathrm{C}$ and $4 \mathrm{MPa}$. These data were average values of three times experiments. The deviation of products was within $4 \%$ of each average value.

The ratio of BOD / ThOD represents the portion of BOD obtained at each condition in the theoretical oxygen demand (ThOD) calculated from identified products at the conditions. The ratio of BOD / ThOD obtained from DCAA was not much different depending on reaction time. On the contrary, the ratio obtained from TCAA varied with the amount of identified product and reaction time. Under all the tested conditions, the obtained ratio was higher than 1.0. It means that some parts of unknown materials were also biodegradable products. Therefore, both identified products and unknown materials could play an important role in the biodegradation of products. After hydrothermal reaction, DCAA and TCAA were not detected even at $7 \mathrm{~min}$. It implies that DCAA and TCAA were partially fractured and then converted to other products at the beginning of hydrothermal reaction. In the total carbon contents of DCAA, $59 \%$ of malic
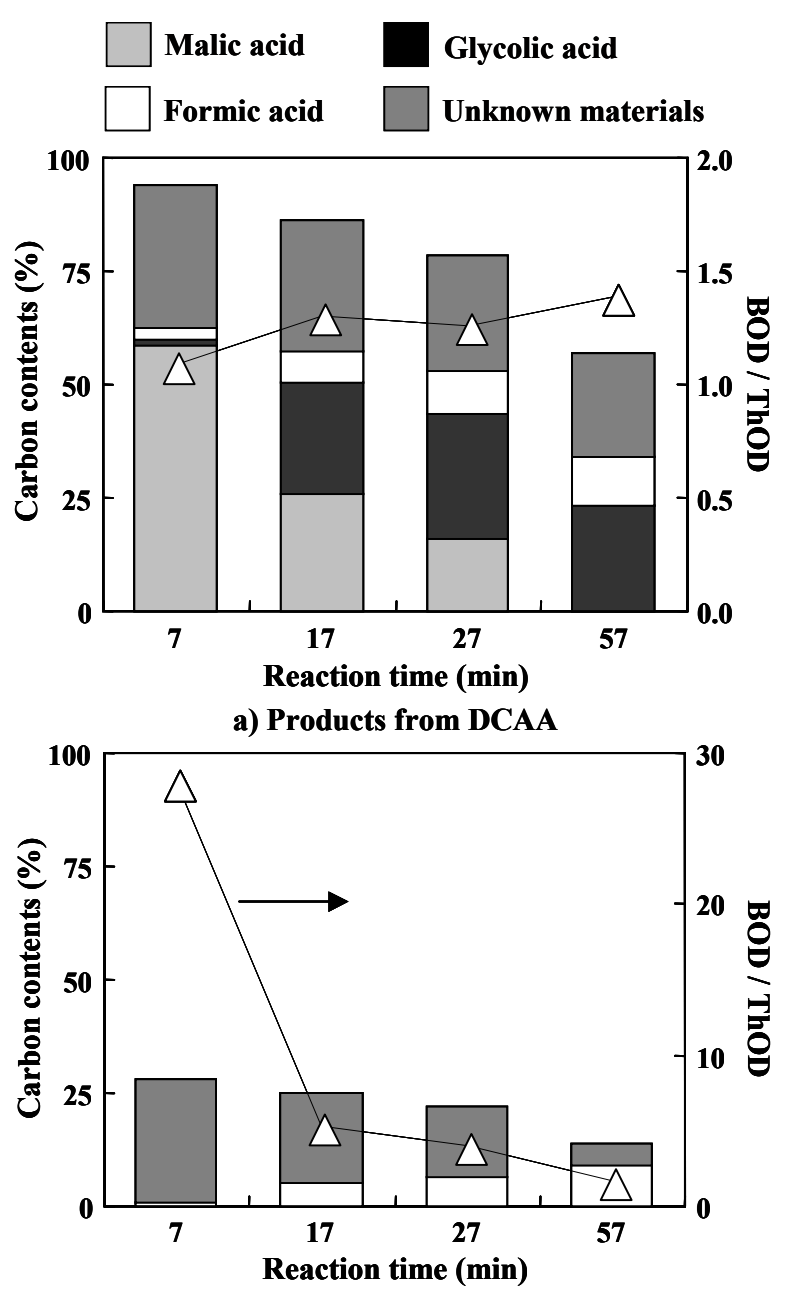

b) Products from TCAA

Figure 1. Effect of reaction time on the content change of products from DCAA and TCAA by hydrothermal reaction at $250{ }^{\circ} \mathrm{C}$ and $4 \mathrm{MPa}$ 
acid, $1 \%$ of glycolic acid and $2 \%$ of formic acid were detected in $7 \mathrm{~min}$. The amount of malic acid decreased, and was converted into more simple organic acids as reaction time increased. Glycolic acid increased up to $28 \%$ at $27 \mathrm{~min}$, and then decreased gradually. However, formic acid increased continuously with increasing reaction time. Finally, $43 \%$ of carbon contents were mineralized at $57 \mathrm{~min}$. In case of TCAA, more than $70 \%$ of total carbon contents were mineralized within 7 min by hydrothermal reaction. Formic acid, which was the only identified product, increased from $1 \%$ in 7 min to $9 \%$ in $57 \mathrm{~min}$. On the contrary, unknown materials reduced from $27 \%$ to $5 \%$ at the same time periods.

\subsection{Reaction mechanism of CAAs under hydrothermal conditions}

The reaction pathway of CAAs under hydrothermal conditions is represented in Figure 2.

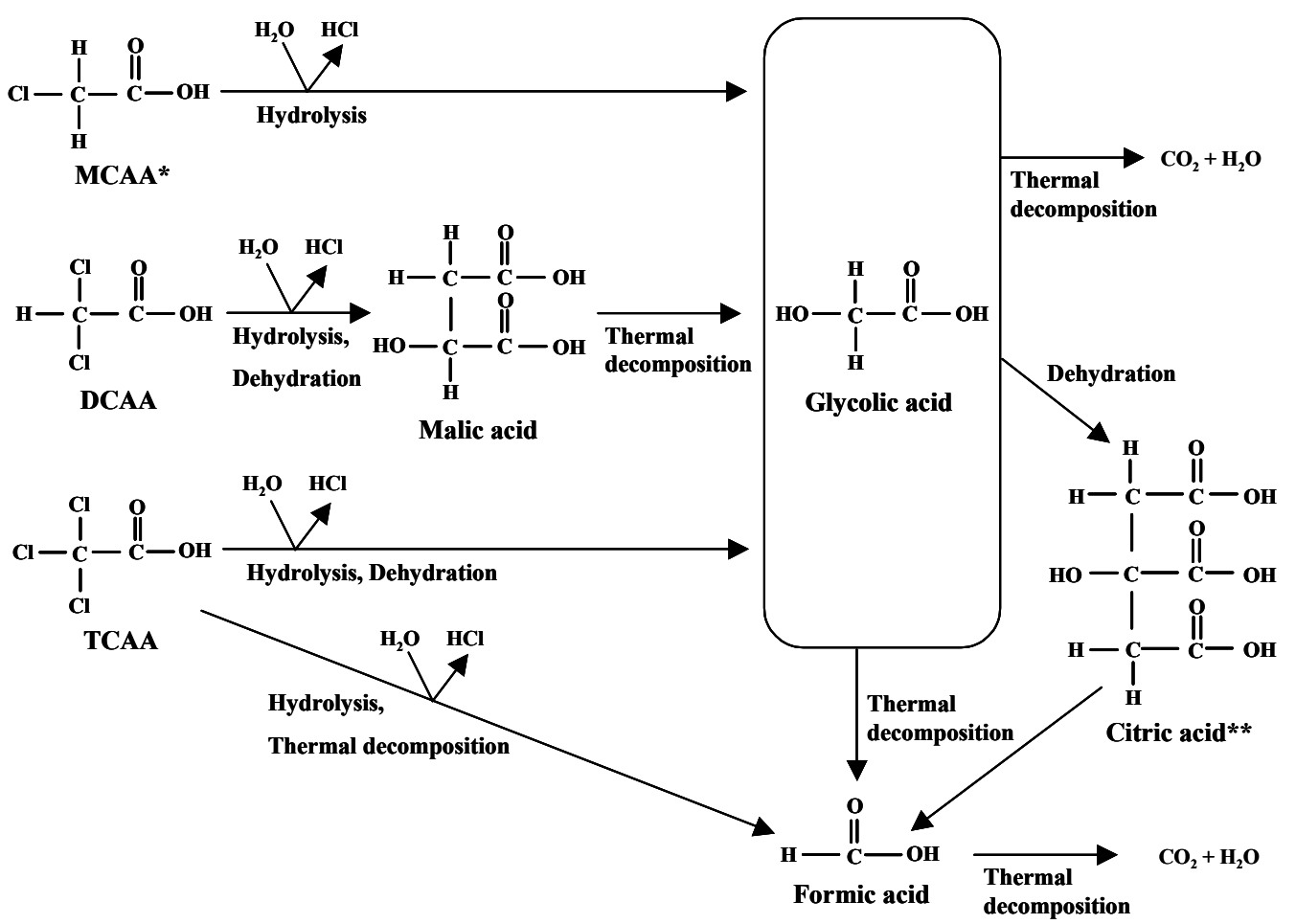

Figure 2. Reaction pathway of CAAs under hydrothermal conditions

*: Previous research results (Kim et al., 2002)

$* *$ : A minor product

In our previous research, partial destruction of MCAA by hydrolysis, production of biodegradable glycolic acid and decomposition of glycolic acid to other organic acids were reported [11]. Based on the results of ion chromatography, attached recalcitrant chlorine atoms of DCAA and TCAA were removed less than $7 \mathrm{~min}$ at the tested conditions. These results imply the production of hydrochloric acid. This HCL originated from removed chlorine atoms by hydrolysis reaction at the beginning of hydrothermal reaction. DCAA was partially fractured and converted to malic acid by hydrolysis and dehydration reaction, then decomposed to glycolic acid by thermal decomposition. After the production of glycolic acid, reaction pathway was not different from that of MCAA. TCAA was rapidly converted to glycolic and formic acid by hydrolysis, dehydration and thermal decomposition. The reaction pathway was similar to that of MCAA. However, formic acid was also produced from hydrolyzed TCAA by thermal decomposition. From these results, only the initial fracture of DCAA and TCAA was different from the reaction pathway of MCAA. The initial decomposition of DCAA and TCAA was mainly due to complex reactions including hydrolysis, dehydration and thermal decomposition. Besides, citric acid, a minor product, was not always detected at all conditions because of its fast decomposition rate under hydrothermal conditions.

The elimination of attached recalcitrant chlorine atoms of CAAs was easily attained at the start of the reaction as shown in section 3.1. Namely, the decomposition of CAAs to glycolic or malic acid is the most important step on the viewpoint of the pretreatment of refractory pollutants prior to conventional biological treatment methods. The decomposition rates of CAAs under hydrothermal conditions are discussed. Figure 3 shows reaction rate constants of CAAs by hydrothermal reaction at $250{ }^{\circ} \mathrm{C}$ and previous 
results of MCAA [11]. Reaction rate constants increased by about 2.3 times with an increase in the number of attached chlorine atoms. Based on these results, it is likely that substituted $-\mathrm{OH}$ parts make hydrolyzed CAAs unstable and easily degradable.

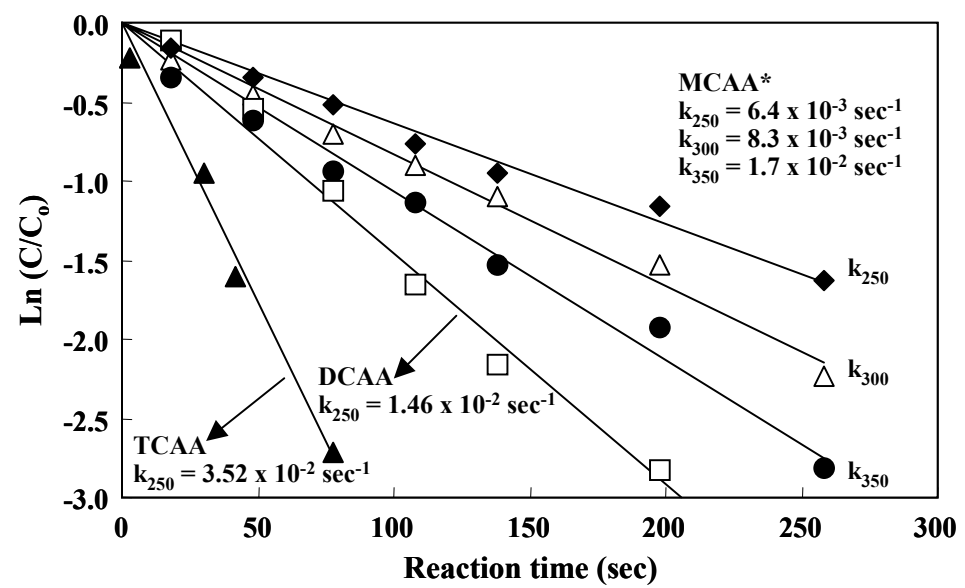

Figure 3. Reaction rate constants of CAAs under hydrothermal conditions

*: Previous research results (Kim et al., 2002)

Thus, the reduction of products increased proportionally with increasing number of attached chlorine atoms of CAAs as shown in section 3.1. This is the unique advantage of hydrothermal reaction compared to other treatment methods. In case of other treatment methods, based on mineralization, required oxidants and reaction time proportionally increased with the contents of refractory materials in a substance. This technology is useful because it has no relation with the concentration of the refractory parts, and it requires only short reaction time.

\subsection{Change of water quality indexes by hydrothermal reaction}

Table 1 shows the change of water quality indexes of di- and trichloroacetic acid by hydrothermal reaction at $250{ }^{\circ} \mathrm{C}$ and $4 \mathrm{MPa}$. Under the conditions, water has unique characteristics comparing to water under normal conditions as shown by Shaw [8] and Savage [9]. Thus, hydrothermal reaction is mainly controlled by various characteristics of water at the specific temperatures and pressures.

Table 1 . Change of water quality indexes of di- and trichloroacetic acid by hydrothermal reaction at $250{ }^{\circ} \mathrm{C}$ and $4 \mathrm{MPa}$

\begin{tabular}{ccccccc}
\hline Specification & $\begin{array}{c}\text { Reaction } \\
\text { time (min) }\end{array}$ & $\begin{array}{c}\text { TOC } \\
(\mathbf{m g} / \mathbf{L})\end{array}$ & $\begin{array}{c}\text { DOC } \\
(\mathbf{m g} / \mathbf{L})\end{array}$ & $\begin{array}{c}\text { BOD } \\
(\mathbf{m g} / \mathbf{L})\end{array}$ & $\begin{array}{c}\mathbf{C O D}_{\mathbf{C r}} \\
(\mathbf{m g} / \mathbf{L})\end{array}$ & $\mathbf{p H}$ \\
\hline \multirow{6}{*}{ DCAA } & - & 1020 & 1020 & 0 & 2550 & 1.6 \\
& 7 & 960 & 960 & 1360 & 2500 & 1.2 \\
& 17 & 880 & 870 & 1460 & 2300 & 1.2 \\
& 27 & 800 & 790 & 1280 & 2100 & 1.2 \\
& 57 & 580 & 580 & 860 & 1500 & 1.2 \\
\hline \multirow{6}{*}{ TCAA } & - & 1000 & 1000 & 0 & 2600 & 1.4 \\
& 7 & 280 & 280 & 390 & 650 & 1.3 \\
& 17 & 250 & 250 & 370 & 550 & 1.3 \\
& 27 & 220 & 220 & 350 & 500 & 1.3 \\
& 57 & 140 & 130 & 210 & 350 & 1.3 \\
\hline
\end{tabular}

Initial BOD values of DCAA and TCAA were lower than detection limits in the laboratory experiments. These results correspond to the results of Ellis [6], including the long induction time and halflife time of CAAs in the field and laboratory study. Initial water quality indexes of DCAA and TCAA did not change in ambient water. Since the deviation of water quality indexes was less than $3 \%$ of each average value, average values of the results of three experiments at each condition were used. The difference between total and soluble contents was not significant under the tested conditions as can be seen from the 
results of TOC and DOC. In case of TCAA, more than $70 \%$ of TOC and $\mathrm{COD}_{\mathrm{Cr}}$ were mineralized within 7 min as shown in Figure 1. After the reaction, $\mathrm{pH}$ values of DCAA and TCAA slightly decreased from 1.6 and 1.4 to 1.2 and 1.3 under all the reaction times. These results correspond with the production of hydrochloric acid at the beginning of hydrothermal reaction as discussed in section 3.1 and 3.2.

\subsection{Relationship between TOC reduction and BOD improvement}

Figure 4 shows the relationship between TOC reduction and BOD improvement of products obtained from CAAs at $250{ }^{\circ} \mathrm{C}$ and $4 \mathrm{MPa}$. In order to know the minimum TOC reduction range to obtain the maximum BOD improvement, the BOD improvement per unit TOC reduction of products at the reaction conditions is discussed.

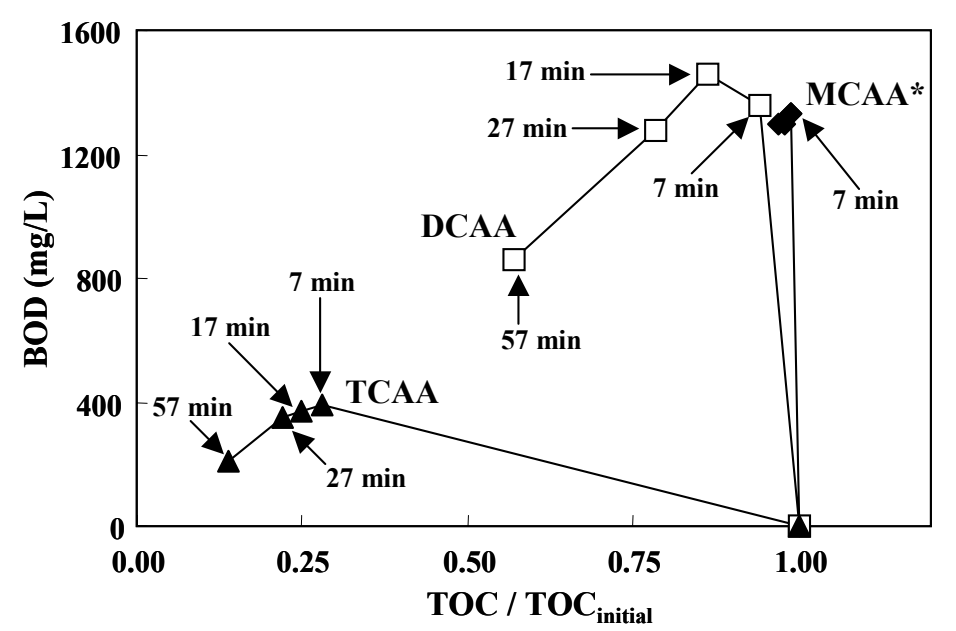

Figure 4. Relationship between TOC reduction and BOD improvement of products obtained from CAAs by hydrothermal reaction at $250{ }^{\circ} \mathrm{C}$ and $4 \mathrm{MPa}$

*: Previous research results (Kim et al., 2002)

In the previous research [11], it was reported that BOD values of products induced from MCAA increased until $1,330 \mathrm{mg} / \mathrm{L}$ in $7 \mathrm{~min}$, then remained in spite of increasing reaction time. In that case, TOC reduction range was less than $4 \%$. In case of DCAA, BOD improvement per unit TOC reduction within 7 min did not vary much. Although there is a slight difference in the initial reaction pathway from that of MCAA, DCAA was converted to biodegradable malic acid in $7 \mathrm{~min}$ by hydrolysis and dehydration reaction. Only $6 \%$ of total carbon content of DCAA was reduced during the structural conversion, as shown in Figure 1 and 2. On the other hand, BOD values decreased after the reaction time of $17 \mathrm{~min}$. This likely happened because thermal decomposition of biodegradable products was faster than the production of biodegradable products by hydrothermal reaction. Finally, BOD value reached $860 \mathrm{mg} / \mathrm{L}$ with reduction of $43 \%$ of total carbon content. In case of TCAA, the decomposition of products reached $72 \%$ of total carbon content of TCAA in $7 \mathrm{~min}$. Since biodegradable products were also decomposed at the same time, the only identified reaction product was formic acid as shown in Figure 1. Thus, obtained BOD values were lower than $400 \mathrm{mg}$ / L. However, the BOD values were significantly higher compared to the initial BOD value of TCAA. From the results of Figure 1, 2 and 4, CAAs were converted to biodegradable products at the beginning of hydrothermal reaction. The production of biodegradable products by structural conversion of CAAs required TOC reduction. However, thermal decomposition of biodegradable products followed by structural conversion, simultaneously induced the reduction of BOD and TOC as reaction time increased. Therefore, to improve BOD, reactions should not significantly reduce TOC after achieving suitable structural conversion. The suitable TOC reduction range was $6 \%$ for DCAA and $72 \%$ for TCAA, respectively. Considering the heat-up time of reactor 1 and the results of DCAA, the required time to obtain BOD values higher than $1,300 \mathrm{mg} / \mathrm{L}$ was within $1 \mathrm{~min}$ after reaching the desired conditions, at the reaction temperature of $250{ }^{\circ} \mathrm{C}$. It implies the possibility of applying continuous hydrothermal treatment method for pretreatment of refractory pollutants.

\subsection{Variation of biodegradability depending on reaction time}


Both the ratio of BOD / TOC and BOD / $\mathrm{COD}_{\mathrm{Cr}}$ represent the biodegradable portion of all organic carbon contents in a substance, and are commonly used to evaluate biodegradability $[\mathbf{1 1 , 1 5}$. In this research, the ratio of $\mathrm{BOD} / \mathrm{COD}_{\mathrm{Cr}}$ was selected as the index of biodegradability. Both identified products and unknown materials could contribute to the change of biodegradability and BOD as represented in figure 1,2 and 4. Thus, biodegradability and the total amount of biodegradable substances in products are investigated. Figure 5 represents the effect of reaction time on the biodegradability improvement of products from DCAA and TCAA.

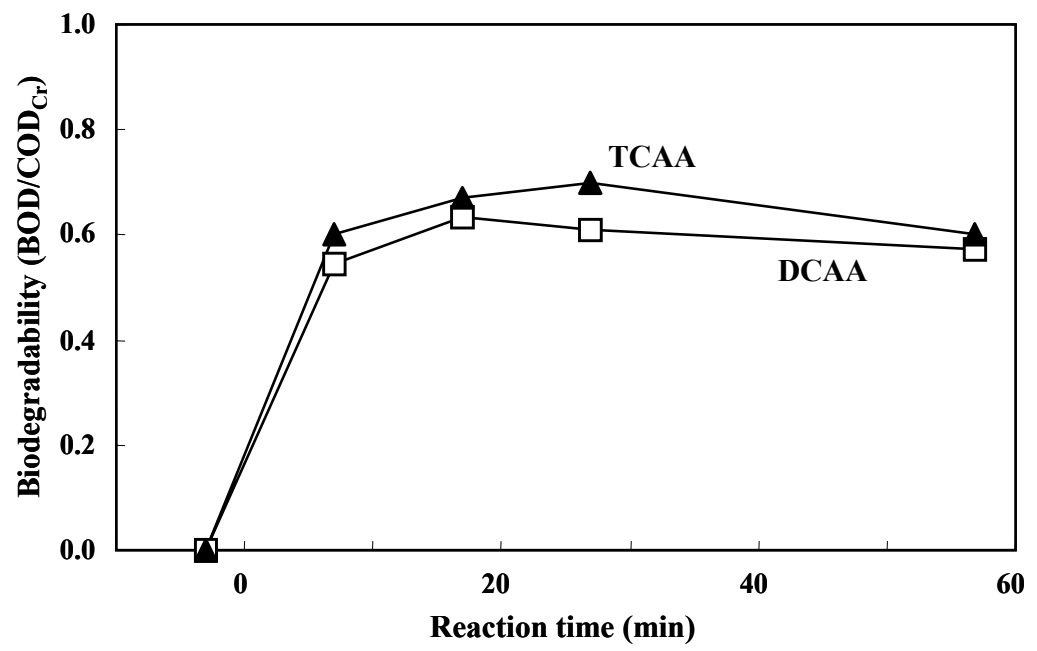

Figure 5. Effect of reaction time on the biodegradability change of products from DCAA and TCAA by hydrothermal reaction at $250{ }^{\circ} \mathrm{C}$ and $4 \mathrm{MPa}$

The initial biodegradability of DCAA and TCAA was almost zero. Under the reaction conditions of $250{ }^{\circ} \mathrm{C}$ and $4 \mathrm{MPa}$, biodegradability of DCAA increased to 0.54 within $7 \mathrm{~min}$, and did not vary much up to reaction time of $57 \mathrm{~min}$. In case of TCAA, the biodegradability change was similar to that of DCAA. It can be explained by the fact that the structural conversion from DCAA and TCAA to biodegradable products occurred at the beginning of hydrothermal reaction, as shown in Figure 1 and 4 . Generally, some substances that have the ratio of $\mathrm{BOD} / \mathrm{COD}_{\mathrm{Cr}}$ higher than 0.4 are usually considered as thoroughly biodegradable materials [15]. The biodegradability values of DCAA and TCAA treated by hydrothermal reaction were suitable values for conventional biological treatment methods. However, the amount of biodegradable carbon contents of treated DCAA was much higher than that of treated TCAA as shown in Figure 1. Therefore, the results of DCAA were more desirable results than those of TCAA on the viewpoint of biodegradability improvement from the structural conversion of refractory pollutants without much reduction of carbon contents. Besides, chlorine ions were present in the reaction products because of the dissociation of hydrochloric acid produced from CAAs by hydrolysis reaction as discussed in section 3.1 and 3.2. From the results of ion chromatography, the concentration of chlorine ions of products obtained from DCAA and TCAA was 3,000 and $4,500 \mathrm{mg} / \mathrm{L}$, respectively. However, the biodegradability of products was not critically retarded at the concentration range of chlorine ions tested in this research.

\section{CONCLUSIONS}

Hydrothermal reaction was investigated to improve biodegradability of refractory pollutants in this research. The elimination of recalcitrant chlorine atoms from CAAs, structural conversion of CAAs and production of biodegradable products were easily achieved by hydrolysis, dehydration and thermal decomposition at $250{ }^{\circ} \mathrm{C}$ and $4 \mathrm{MPa}$ within $1 \mathrm{~min}$. The extremely short reaction time could make this technology the most alternative method from any other treatment methods. During hydrothermal reaction, the decomposition rate constants of CAAs increased around 2.3 times with an increase in the number of attached chlorine atoms, regardless of the concentration of refractory parts in CAAs. The results could be applied as basic data to the treatment of Chlorinated refractory pollutants composed of linear hydrocarbon structure under hydrothermal conditions. The biodegradability of products was not fatally retarded by the presence of chlorine ions under the tested conditions. In order to avoid the thermal decomposition of biodegradable products, reactions must be adjusted to obtain the minimum TOC reduction range enough for 
the improvement of BOD. Although these results obtained from batch experiments, hydrothermal reaction might be possibly applied to continuous treatment method for pretreatment of refractory pollutants, due to very simple apparatus, easy control and short reaction time with exception of heat-up time. The feasibility of hydrothermal reaction was found in case of the pretreatment of refractory pollutants such as CAAs for the following conventional biological treatment methods. For future, further inspection of hydrothermal reaction is also required to clarify reaction mechanism of other kinds of refractory pollutants and to apply this technology to real wastewater.

\section{ACKNOWLEDGMENTS}

The authors are grateful for the financial support provided by Industrial Technology Research Grant Program in 01A42017c, from New Energy and Industrial Technology Development Organization (NEDO) of Japan, and a research grant (A02-13) in Ecological Engineering for Homeostatic Human Activity from The 21st Century COE Program, the Ministry of Education, Culture, Sports, Science and Technology (Japan).

\section{REFERENCES}

1. Van Agteren, M. H., Keuning, S. and Janssen, D. B., Handbook on biodegradation and biological treatment of hazardous organic compounds, Kluwer academic publishers, Dordrecht, The Netherlands, pp. 77-187 (1998).

2. Sarzanini, C., Bruzzoniti, M. C. and Mentasti, E., Preconcentration and separation of haloaceticacids by ion chromatography. J. Chromatogr. A., 850, 197-211 (1999).

3. McCulloch, A., Trichloacetic acid in the environment. Chemosphere, 47, 667-687 (2002).

4. Kühn, R. and Pattard, M., Results of the harmful effects of water pollutions to green algae (scenedesmus subspicatus) in the cell multiplication inhibition test. Water Res., 24, 31-38 (1990).

5. Tao, L., Kramer, P. M., Ge, R. and Pereira, M. A., Effect of dichloroacetic acid and trichloroacetic acid on DNA methylation in liver and tumors of female B6C3F1 mice. Toxicol. Sci., 43, 139-144 (1998).

6. Ellis, D. A., Hanson, M. L., Sibley, P. K., Shahid, T., Fineberg, N. A., Solomon, K. R., Muir, D. C.G. and Mabery, S. A., The fate and persistence of trifluoroacetic and chloroacetic acids in pond water. Chemosphere, 42, 309-318 (2001).

7. Raymond, J. W., Rogers, T. N., Shonnard, D. R. and Kline, A. A., A review of structure-based biodegradation estimation methods. J. Hazard. Mater., B84, 189-215 (2001).

8. Shaw, R. W., Bill, T. B., Clifford, A. A., Eckert, C. A. and Frank, E. U., Supercritical water; a medium for chemistry. Chem. Eng. News, 12, 26-39 (1991).

9. Savage, E., Organic chemical reactions in supercritical water. Chem. Res., 99, 603-621, (1999).

10. Kim, K., Fujita, M., Daimon, H. and Fujie, K., Biodegradability Improvement and Structural Conversion of Polyvinyl Alcohol (PVA) by Sub- and Supercritical Water Reaction. J. Chem. Eng. Jpn., submitted for publication (2003).

11. Kim, K., Fujita, M., Daimon, H. and Fujie, K., Change of Monochloroacetic acid to Biodegradable Organic Acids by Hydrothermal Reaction. J. Hazard. M., submitted for publication (2002).

12. Quitain, A. T., Sato, N., Daimon, H. and Fujie, K., Production of valuable materials by hydrothermal treatment of shrimp shells. Ind. Eng. Chem. Res., 40, 5885-5888 (2001).

13. Kang, K., Quitain, A. T., Daimon, H., Noda, R., Goto, N., Hu, H.-Y. and Fujie, K., Optimization of amino acids production from waste fish entrails by hydrolysis in sub- andsupercritical water. Can. J. Chem. Eng., 79, 65-70 (2001).

14. Xie, Y., Analyzing haloacetic acids using gas chromatography/mass spectrometry. Water Res., 35, 1599$1602(2001)$.

15. Yu, C. P. and Yu, Y. H., Identifying useful real-time control parameters in ozonation process. Water Sci. Technol., 42, 435-440, (2000). 